\section{Les collectifs \\ d'usagers dans les champs du sida et de la toxicomanie}

$>$ L'ampleur de la mobilisation collective suscitée par l'épidémie de sida a été soulignée maintes fois. En raison d'une logique de diffusion sélective, l'infection par le virus de l'immunodéficience humaine (VIH) n'a pas seulement déclenché l'action des malades et de leurs proches, comme c'est habituellement le cas dans le domaine des maladies, mais aussi celle des deux «groupes sociaux » les plus affectés : les homosexuels masculins et les usagers de drogues. Existant depuis près d'un siècle, les collectifs de «malades » recouvrent des configurations diversifiées, qui vont des groupes consensuels se développant dans les années 1930 aux groupes plus contestataires émergeant au cours des années 1970. Nous montrerons ici comment, dans les champs du sida et de la toxicomanie, ces collectifs d'usagers se sont multipliés au travers de choix d'identifications publiques différenciées. Dans le domaine de la lutte contre le sida, coexistent toutes les formes de mobilisation que l'on trouvait déjà préalablement dans le champ des maladies. Dans le domaine de l'usage de drogues, les logiques d'action sont moins diversifiées et se partagent en deux grandes catégories : groupes d'intérêt et self-help groups (groupes d'entraide). <

\section{Maladies et mobilisation collective}

Historiquement, la mobilisation sociale relative aux maladies connaît trois grandes périodes avant l'apparition du sida [1]. La première, qui s'ouvre au XIxe siècle, est celle de la charité. À partir de la fin du xixe et du début du xxe siècle, la lutte contre la maladie s'intègre à celle contre les fléaux sociaux : apparaissent alors les

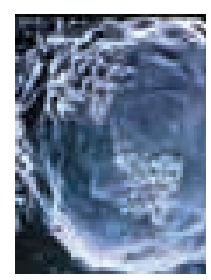

\section{Broqua : Centre} d'ethnologie française, Musée national des arts « ligues » contre la tuberculose, la syphilis, l'alcoolisme ou le cancer. Cette nouvelle approche repose sur une forte distance entre les malades et ceux qui s'occupent d'eux : «femmes du monde », médecins, notables. La troisième et traditions populaires. M. Jauffret-Roustide : Centre de recherches psychotropes, santé mentale, société (CNRS-Paris V), Institut de veille sanitaire, 12, rue du Val d'Osne, 94415 Saint-Maurice cedex, France m.jauffret@invs.sante.fr période se caractérise par une plus grande diversité des logiques d'action, que l'on peut répartir en trois grandes catégories : I'activité de collecte de fonds et d'aide à la recherche, la gestion de structures de prise en charge et, enfin, les regroupements d'usagers, marqués par le développement du concept de self-help (autosupport) aux États-Unis à partir des années 1970 $[2,3]$.

Ainsi, des « groupes de malades » existaient déjà avant les années 1980, dont les caractéristiques et les finalités préfigurent celles des associations de lutte contre le sida. La mobilisation concerne de nombreuses maladies, souvent chroniques ou dégénératives (tuberculose, diabète, hémophilie, myopathie, mucoviscidose ou encore cancer). Même si le phénomène ne prend un essor réel que dans les années 1960-1970, les premiers ont été constitués dès le début du siècle : blessés du poumon (1921), diabétiques (années 1930), hémophiles (années 1950). Ces groupes, dont le monde médical constitue le principal interlocuteur, se structurent autour de deux orientations majeures : la prise en charge autonome du mal, qui va jusqu'à l'acquisition d'un savoir scientifique et technique rivalisant avec 
celui du médecin et conférant au malade le statut d' «autosoignant » [4], et l'élaboration d'une identité collective autour du trait commun de la maladie.

Les premiers travaux sur le self-help apparaissent au moment où sont créés les Alcoholics anonymous (AA), en 1935, aux États-Unis. Ce type de groupe émerge en réaction aux carences du système institutionnel de prise en charge des alcooliques, perçu comme bureaucratique, peu efficace et culpabilisant pour les «patients ». AA met en place des groupes de parole et de soutien au cours desquels les alcooliques échangent leur expérience de ce qu'ils nomment la «maladie alcoolique ». En France, cette reconnaissance de la centralité de l'expérience des malades avait déjà été mise en avant dans le cadre des Sociétés de secours mutuel, à la fin du XIXe siècle, ou avec l'Association des blessés du poumon, après la Première Guerre mondiale [5]. Entre les années 1930 et 1950 se développent des associations liées aux maladies chroniques. Elles font valoir une forme de délégation inversée entre le patient et son médecin, ainsi qu'une possible gestion de la maladie par le malade. Elles mettent également en place des groupes de thérapie collective pour les malades et leurs proches. Jusque dans les années 1970, ces collectifs postulent que les stigmatisés doivent s'adapter au monde des «normaux ».

Dans les années 1970, se produit un tournant fondamental avec l'émergence de nouveaux types de collectifs plus contestataires, qui utilisent des répertoires d'action plus agressifs, remettent en cause l'ordre social et perçoivent en la médecine une volonté de les contrôler et de les normaliser. Ces nouveaux collectifs n'entrent plus dans la catégorie des groupes thérapeutiques; ils peuvent être qualifiés de groupes politiques, dont l'objectif n'est plus la conformation des stigmatisés aux normes sociales dominantes, mais la transformation de ces normes afin de rendre la société plus tolérante. Ces nouveaux groupes de self-help s'inscrivent dans une double logique : celle du militantisme et celle du consumérisme. La lutte contre la stigmatisation dont les malades sont victimes occupe ici une place centrale, sous la forme d'une revendication du droit à faire valoir leur expertise et leur participation aux politiques publiques les concernant. Ces collectifs marquent la fin de l'idéologie de l'assimilation et de la réhabilitation des groupes stigmatisés [6].

Dans la littérature anglo-saxonne, différents auteurs se sont efforcés de catégoriser les associations de malades soit selon leurs objectifs, en distinguant alors le modèle de l'association philanthropique de celui des maladies chroniques ou de l'association plus politique, soit selon leurs rapports aux professionnels, distinguant l'association-auxiliaire, l'association-partenaire et l'association-opposante [7]. En France, ces dernières années, seuls quelques chercheurs se sont intéressés aux collectifs de malades - tels que l'Association française contre les myopathies, en particulier dans leur rapport à la recherche scientifique [8]. Mais c'est à propos de l'épidémie de sida que ces travaux ont été les plus nombreux, soit sur des associations créées antérieurement, mais prenant part à la lutte contre le sida, comme l'Association française des hémophiles [9], soit sur des associations créées dans le contexte de l'épidémie [1015].

\section{Les associations de lutte contre le sida}

C'est en 1981 que sont identifiés les premiers cas de sida. Aux États-Unis, contrairement à ce qui se passera en France, des « groupes de malades » apparaissent dès 1982, c'est-à-dire la même année que la première « organisation de service » créée à New York : la Gay men's health crisis. La création des premiers groupes, à San Francisco et à New York, est déclenchée ou favorisée par des médecins qui décident de mettre leurs malades en relation afin qu'ils s'organisent sur le mode du self-help. Rapidement, toutefois, la mobilisation prend un tour plus militant, l'héritage des mouvements homosexuels jouant ici un rôle fondamental. En 1983, le AIDS Network est créé à New York, fédérant des homosexuels, atteints ou non par le sida, que les seules logiques de service ou d'autosupport laissent insatisfaits, et qui souhaitent développer une action revendicatrice et politique ; ils organisent notamment une première manifestation en avril à l'occasion d'une conférence sur le sida. D'autres manifestations ont également lieu les mois suivants dans plusieurs villes américaines, qui puisent tant au registre de la commémoration publique qu'à celui de la contestation sociale. Au mois de juin 1983 se déroule à Denver le second forum national sur le sida, au cours duquel des représentants des groupes de malades rédigent une charte de principes qualifiée depuis de «Principes de Denver », dont Act UpParis écrira plus tard qu'ils « représentent l'acte de naissance de l'activisme sida » et « décrivent le programme de l'activisme sida pour les années qui suivent » [16]. Ainsi, moins de deux années après l'apparition des premiers cas, existent déjà aux États-Unis des groupes mués par une logique de service communautaire, de self-help ou de contestation politique, tout ceci (fait important) dans le cadre d'une mobilisation exclusivement homosexuelle.

Il en va très différemment en France, où la mobilisation est beaucoup plus tardive, et dans un premier temps moins diversifiée. En 1982, les malades recensés sont peu nombreux ; leur cas suscite pourtant l'inquiétude de quelques médecins qui constituent cette année-là le Groupe français de travail sur le sida, et tentent en vain de susciter la mobilisation des mouvements homosexuels. C'est en août 1983 qu'un militant issu de ces mouvements, Patrice Meyer, crée la première association française du genre : Vaincre le sida (VLS). Mais en raison des microconflits qui scandent son évolution, ce groupe ne connaît pas au cours des premières années l'essor de ses successeurs. II est pourtant celui qui, avant tous les autres, établit les principes de l'action communautaire et de la lutte contre la maladie, en 
produisant des brochures d'information, en ouvrant une ligne d'écoute téléphonique durant l'hiver 1984 et en mettant en place les premières formes d'aide aux malades.

L'association AIDES, qui fait son apparition un an plus tard, connaîtra à l'inverse un essor considérable qui la placera longtemps en tête du mouvement associatif de lutte contre le sida en France. Après la mort de son ami Michel Foucault dans des circonstances qu'il déplore, le sociologue Daniel Defert décide de mettre en place un groupe de réflexion sur la pratique médicale face au sida, dont il a éprouvé la « faillite », notamment au travers de la dissimulation du diagnostic. Les statuts de AIDES sont officiellement déposés en décembre 1984, soit trois années après l'identification des premiers cas de sida, un an après la découverte de l'agent viral (VIH), mais plusieurs mois avant la mise au point des tests de dépistage qui donneront naissance à la figure du «séropositif », et permettront dès lors de mieux visualiser l'épidémie. L'association définit alors ses objectifs autour du personnage du malade, après l'échec d'un premier projet plus spécifiquement tourné vers les homosexuels, adoptant même d'emblée une attitude hostile à toute identification à I'homosexualité. Au cours des années 1985 et 1986, alors que de plus en plus de personnes (en particulier chez les homosexuels masculins) prennent connaissance de leur statut sérologique, l'action de AIDES peut se développer sur les deux principaux fronts de l'aide aux malades et de la prévention.

Le développement de l'association ne se fait pas sans heurt, et l'année 1987 se trouve marquée par une scission au sein du conseil d'administration. Le conflit porte en grande partie sur la posture que doit adopter - et la place que doit occuper - le volontaire, en particulier sur la distinction (et la distance) à établir entre ce dernier et l'« usager » de l'association. II se traduit par la démission en mars 1987 de plusieurs membres, dont les cofondateurs Frédéric Edelmann et Jean-Florian Mettetal, qui rejoignent l'association ARCAT-Sida (Association de recherche, de communication et d'action pour l'accès aux traitements), créée deux ans plus tôt par un médecin dans le but d'optimiser le suivi des malades grâce à un soutien à un groupe de cliniciens et de chercheurs. Avec de nouveaux statuts déposés début 1988 et la création du Journal du sida en 1989 ainsi que celle du «Point Solidarité », l'association va développer ses activités sur un triple champ d'intervention largement professionnalisé : l'information sur le sida, le soutien au monde médical et l'action sociale en direction des personnes atteintes les plus marginalisées.

À partir des années 1987-1988, le champ associatif de lutte contre le sida connaît un important développement, avec la création de multiples associations plus spécialisées. La grande majorité reste fidèle au principe de AIDES visant à se distancier de toute référence à l'homosexualité. Mais les années 1988 1989 voient aussi apparaître des associations au caractère plus militant : Différence... Positif, Positifs, Solidarité Plus et surtout Act Up-Paris vont imposer une nouvelle présence, se fai- sant explicitement la voix des séropositifs et, pour Act Up, des homosexuels. Plus largement, cette période est également celle où émerge avec force (et sous différentes formes), dans l'espace public, une expression du vécu de la séropositivité, de la maladie et du deuil. L'apparition d'Act Up-Paris, qui deviendra le fer de lance d'une nouvelle génération associative, résulte ainsi de la conjonction de deux phénomènes émergents : l'expression nouvelle d'une parole publique des personnes atteintes par le VIH et l'inclusion d'une revendication identitaire homosexuelle à la lutte contre le sida, ces deux dimensions ne trouvant pas leur place au sein des principales associations créées antérieurement. C'est également dans une logique d'opposition à leurs aînées que les trois autres associations de cette nouvelle génération créent en novembre 1990 le Syndicat des personnes atteintes par le sida (SPAS), récusant ainsi jusqu'au statut associatif.

Au cours de la seconde décennie de l'épidémie, l'espace de la lutte contre le sida se stabilisera en s'organisant autour de deux pôles majeurs (réformiste contre révolutionnaire) incarnés par AIDES et Act Up. II aura donc fallu près d'une décennie pour qu'apparaisse en France la diversité des formes de mobilisation observable dans la seule ville de New York dès les deux premières années de l'épidémie.

II n'en reste pas moins que la particularité de la mobilisation contre le sida est d'englober l'ensemble des formes de collectifs qui existent dans le champ des maladies, de manière généralement plus exclusive : l'action caritative, l'autosupport, la contestation politique, la récolte de fonds pour la recherche... D'où l'importance des identités publiques choisies par les associations, qui ont toutes à se positionner les unes par rapport aux autres. Ces identifications posent le plus souvent la question de la place des malades, d'une part, et des homosexuels, d'autre part, dans la définition qui est donnée de la mobilisation.

\section{Les groupes d'usagers de drogues}

Concernant les usagers de drogues, l'autosupport occupe une place plus marginale en France : seules deux associations émergent, dont l'une ne regroupe que quelques dizaines d'usagers [17-19]. Dans ce domaine, deux types de groupes peuvent être distingués, les groupes d'intérêt (interest groups) et les groupes d'entraide (self-help groups) [20]. Autosupport des usagers de drogues (ASUD), créé en 1992, représente des groupes d'intérêt ayant surtout un objectif politique de défense des droits des usagers et fonctionnant comme des syndicats, avec des revendications contestataires vis-à-vis des politiques de la drogue et de la toxicomanie. Narcotiques anonymes (NA), association créée en 1984, répond quant à elle à la logique des groupes d'entraide, plus consensuels, qui affichent des objectifs de soutien entre usagers de drogues et fonctionnent comme des groupes de parole. Si les membres de NA se désignent et se 
considèrent comme des malades (atteints de la maladie de la dépendance), les militants d'ASUD s'insurgent au contraire contre cette forme de catégorisation et font valoir que l'usage de drogues n'est pas une maladie, mais un choix. Tous ces groupes d'autosupport sont dotés d'une double spécificité, tout d'abord parce que l'usage de drogues n'est pas assimilé à une maladie, ensuite parce que le trait commun qui pousse les usagers à se rassembler (la consommation de drogues) est répréhensible par la loi. Si les groupes d'autosupport d'usagers de drogues ne sont donc pas assimilables aux collectifs de «patients » ou aux associations de «malades », ils participent d'un même processus d'autonomisation.

En France, le premier groupe d'usagers de drogues est donc NA, qui voit le jour en 1984 à l'initiative de quelques usagers de drogues anglo-saxons devenus abstinents, et souhaitant retrouver les réunions qu'ils fréquentaient aux États-Unis. La première réunion de NA en langue française a lieu à Paris, le groupe est alors constitué de quelques personnes; aujourd'hui, 20 ans après, une quarantaine de réunions se tiennent à Paris chaque semaine. Aux États-Unis, ces groupes ont une autre ampleur, puisque plusieurs milliers de réunions sont proposées à New York chaque semaine.

Les premiers groupes NA ont été créés en 1953 aux États-Unis par des membres des AA qui ne parvenaient plus à s'identifier aux alcooliques [21]. La «théorie de la dépendance » mise en avant par NA est plus large que celle proposée par AA, car si AA propose une aide pour arrêter de boire de l'alcool, NA propose à ses membres une abstinence de « tout produit modifiant le comportement », c'est-à-dire non seulement des substances illicites telles que l'héroïne, la cocaïne ou le cannabis, mais aussi des substances licites telles que l'alcool ou les médicaments. À NA, ce n'est pas le produit consommé qui compte mais la dépendance, qui réduirait l'usager de drogues à une forme d'asservissement et dont NA propose de se libérer par l'abstinence, les groupes de parole et l'entraide des pairs.

À l'instar de AA dont la création est liée aux groupes 0xford, un mouvement évangélique axé sur le relèvement spirituel des alcooliques, le discours de NA est empreint de spiritualité. NA est régi par des principes «spirituels » (démocratie, volontariat, autonomie et anonymat). Douze «traditions » définissent les règles de fonctionnement des groupes, et douze «étapes» indiquent aux individus la voie vers l'abstinence et le « rétablissement ». Parmi ces «traditions », la dixième prône l'interdiction de tout discours public et de relations publiques officielles de l'association avec des partenaires : «NA n'a aucune opinion sur des sujets extérieurs; c'est pourquoi le nom de NA ne devrait jamais être mêlé à des controverses publiques ». L'anonymat est également une règle primordiale qui prévaut à l'intérieur même de l'association, puisque les membres se présentent et se désignent entre eux uniquement par leur prénom, mais aussi à l'extérieur, et plus particulièrement dans les rapports avec les médias, car il est impossible pour un membre de NA de parler au nom du groupe, chacun n'étant autorisé à s'exprimer qu'au nom de sa propre expérience. L'anonymat est justifié par la volonté de protéger le groupe des éventuelles « rechutes » dans l'usage de drogues dont ses membres pourraient être victimes et qui terniraient l'image du groupe, mais aussi de protéger les individus de la valorisation que leur médiatisation pourrait leur apporter, l'«humilité» étant au cœur du programme NA.

ASUD, le premier groupe d'autosupport de type groupe d'intérêt ${ }^{1}$, est créé par Philippe Marchenay, Abdalla Toufik ainsi que Gilles et Phuong Charpy en 1992, huit ans après NA et dans un contexte très différent. Les groupes d'intérêt d'usagers de drogues se constituent dans le sillage des associations issues de la lutte contre le sida, autour du modèle du « malade réformateur », de la mise à disposition de produits de substitution pour les usagers de drogues et du bouleversement des politiques publiques en matière de toxicomanie. En effet, après l'apparition du sida, le champ de la toxicomanie est soumis à un changement paradigmatique fondamental : le champ autrefois régi par le sevrage et la psychothérapie est alors dominé par un nouveau concept, celui de la réduction des risques, qui se construit en France autour de l'accès aux seringues, à la substitution et aux soins pour les usagers de drogues, et qui postule la participation des acteurs concernés, en l'occurrence les usagers de drogues, à la définition des politiques publiques. Contrairement à NA, ASUD ne prône pas l'anonymat de ses membres, car l'usage de drogues est considéré comme un choix qu'il s'agit de pouvoir revendiquer publiquement. Et, collectivement, ASUD ne garde pas le silence, mais prend position sur la question des drogues en France. Dès sa création, le groupe adopte un répertoire rhétorique axé autour de la dénonciation de la situation des usagers de drogues. Le Manifeste d'ASUD (première plate-forme politique du groupe qui sera complétée ensuite par les « 10 mesures ») dénonce le taux important de contamination des usagers de drogues par le virus du sida, la clandestinité et la marginalisation de ces usagers de drogues, et accuse la prohibition d'en être responsable.

Afin d'améliorer la situation des usagers de drogues, ASUD propose la mise en place de la politique de réduction des risques et l'arrêt du régime de prohibition des drogues. Depuis le début, c'est à partir d'une problématique sanitaire qu'ASUD se positionne dans le champ politique autour des questions de la citoyenneté et des droits des usagers de drogues. Ce positionnement du groupe dans le champ sanitaire lui permet d'obtenir un financement de divers organismes publics, mais il tend à donner l'impression que les actions de l'association restent figées autour de la question sanitaire : elles se concentrent d'ailleurs prioritairement autour de la question de la prévention du sida et des hépatites. Aujourd'hui encore, les actions

Au milieu des années 1990, d'autres groupes d'intérêt constitués d'usagers de drogues ont existé, mais de manière très éphémère (parfois quelques mois) et n'ont regroupé qu'une poignée d'usagers de drogues. 
plus politiques autour du cadre légal ne constituent pas l'aspect le plus visible d'ASUD. Le répertoire d'action collective du groupe ASUD reste très conventionnel en comparaison de celui de mouvements activistes comme Act Up. ASUD utilise des moyens plus modérés tels que la création d'un journal, qui constitue le vecteur principal de communication et de visibilité pour le groupe, la participation aussi bien à des réunions de collectifs contestataires (Limiter la casse, CAL 70) qu'à des réunions institutionnelles organisées par la division Sida ou la division Toxicomanie de la Direction générale de la Santé (DGS) ou par la Mission interministérielle de lutte contre la drogue et la toxicomanie (MILDT), et l'organisation de manifestations réclamant l'ouverture du débat sur la question des drogues. Ce répertoire évolue peu et les stratégies d'action restent très homogènes.

Ainsi, les prises de parole publiques répondent à des normes bien précises dans chacun des deux groupes d'usagers de drogues. À NA, c'est le témoignage personnel et interne au groupe qui est promu, car chaque dépendant est sensé avoir vécu et vivre encore les mêmes expériences que ses pairs. À ASUD, au contraire, c'est un discours politique et collectif sensé représenter l'association et être le porte-parole de l'ensemble des usagers de drogues qui est valorisé, sans jamais passer par le témoignage personnel, considéré comme humiliant.

\section{Conclusions}

Au fond, deux dimensions liées entre elles font la particularité des mouvements d'usagers dans les domaines du sida et de la toxicomanie. La première concerne la pluralité des postures adoptées, l'une étant presque inédite dans la lutte contre les maladies en France : la contestation sociale et politique (ou encore l'activisme). La seconde est relative aux enjeux de définition de la cause que défendent les groupes, dont dépend aussi la définition qu'ils donnent d'eux-mêmes. La qualité de malade ou de patient est alors bien souvent rejetée au profit d'autres figures, qui visent en grande partie à convertir en identité sociale valorisée les qualités stigmatisées et infériorisées des populations concernées. $\diamond$

\section{SUMMARY}

Collective of users in the fields of AIDS and drug addiction

The extent of mobilization caused by the epidemy of AIDS was underlined many times. Because of a logic of selective dissemination, infection by HIV not only started action of patients and their close relations, but also that of the two most affected social groups : homosexual men and drug users. Existing since almost a century, the collectives of patients have shown various configurations, from consensual groups which developped in the thirties to protestor groups emerging within the seventies. We show here how both in AIDS and drug addiction, the collectives of users multiplied through differentiated public identifi- cation choices. In the fight against AIDS, all the forms of mobilization coexist. Concerning drugs usage, actions are less diversified and divide in two main categories: groups of interest and self-help groups. $\diamond$

\section{RÉFÉRENCES}

1. Pinell P. Pour une étude du mouvement associatif : éléments d'analyse sociologique. Gestions Hospitalières 1997 ; 368 : 498-504.

2. Katz AH, Bender $\varepsilon$ I. Self-help groups in Western society : history and prospects. J Appl Behav Sci 1976; 12 : 265-82.

3. Katz AH. Self-help health groups: some clarifications. Soc Sci Med 1979 ; 13 A : 491-4.

4. Herzlich C, Pierret J. Malades d'hier, malades d'aujourd'hui : de la mort collective au devoir de guérison. Paris : Payot, 1991 : 314 p.

5. Herzlich C. Les difficultés de constitution d'une cause. Sciences Sociales et Santé $1995 ; 13: 39-44$.

6. Anspach R. From stigma to identity politics: political activism among the physically disabled and former mental patients. Soc Sci Med 1979; 13A : 765-73.

7. Rabeharisoa V, Callon M, Demonty B. Les associations de malades et la recherche. I. Des self-help groups aux associations de malades. Med Sci (Paris) 2000 ; $16: 945-9$.

8. Rabeharisoa V, Callon M, Demonty B. Les associations de malades et la recherche. II. Les formes d'engagement des associations de malades dans la recherche en France. Med Sci (Paris) 2000 ; 17 : 1225-31.

9. Carricaburu D. L'hémophilie au risque de la médecine : de la maladie individuelle à la contamination collective par le virus du sida. Paris : Anthropos, $2000: 246$ p.

10. Pollak M. Constitution, diversification et échec de la généralisation d'une grande cause : le cas de la lutte contre le sida. Politix 1991; 16 : 80-90.

11. Fillieule 0, Broqua C. Les associations de lutte contre le sida : approche des logiques de l'engagement à AIDES et à Act Up. Paris : CEVIPOF/MIRE, $2000: 272 \mathrm{p}$.

12. Broqua C, Fillieule 0. Trajectoires d'engagement : AIDES et Act Up. Paris : Textuel, $2001: 94 \mathrm{p}$.

13. Broqua C, Fillieule 0. La lutte contre le sida. In : Crettiez X, Sommier I, eds. La France rebelle : tous les foyers, mouvements et acteurs de la contestation. Paris : Éditions Michalon, 2002 : 329-43.

14. Barbot J. Les malades en mouvements : la médecine et la science à l'épreuve du sida. Paris : Balland, 2002 : $308 \mathrm{p}$.

15. Pinell $\mathrm{P}$, Broqua $\mathrm{C}$, de Busscher $\mathrm{PO}$, et al. Une épidémie politique : la lutte contre le sida en France (1981-1996). Paris : PUF, $2002: 416 \mathrm{p}$.

16. Act Up-Paris. Le sida. Paris : Éditions Dagorno, $1994: 436$ p.

17. Jauffret M. L'autosuppport des usagers de drogues en France : groupes d'entraide et groupes d'intérêt. Documents du Groupement de Recherche Psychotropes, Politique et Société $2000 ; 6: 88 \mathrm{p}$.

18. Jauffret $M$. La réduction des risques : enjeux autour d'une mobilisation collective. Mana $2001 ; 8$ : 161-88.

19. Jauffret-Roustide M. Les mouvements contre la prohibition des drogues. In : Crettiez X, Sommier I, eds. La France rebelle : tous les foyers, mouvements et acteurs de la contestation. Paris : Éditions Michalon, 2002 : 511-6.

20. Trautmann F. Peer support as a method of risk reduction in injecting drug-user communities : experiences in dutch project and the « European peer support project 》. J Drug Issues $1995 ; 25: 617-28$.

21. Wells B. Narcotics anonymous in Britain. In : Strang J, Gossop M, eds. Heroin addiction and drug policy: The British system. Oxford-New York : Oxford University Press, $1994: 240-7$
TIRÉS À PART

M. Jauffret-Roustide 